\title{
Metabolic Clearance of Biologically Active Luteinizing Hormone in Man
}

\author{
Johannes D. Veldhuis, Franco Fraioli, Alan D. Rogol, and Maria L. Dufau \\ Departments of Internal Medicine, Pharmacology, and Pediatrics, University of Virginia School of Medicine, Charlottesville, Virginia \\ 22904; Department of Medicine, Clinica Medica V, Rome, Italy; Molecular Endocrinology Section, Endocrinology and Reproduction \\ Research Branch, National Institute of Child Health and Human Development, Bethesda, Maryland 20892
}

\begin{abstract}
The plasma metabolic clearance of biologically active luteinizing hormone (bioactive LH) was studied using the rat interstitial cell testosterone (RICT) bioassay in six hypogonadotropic men after single bolus injection of highly purified human $\mathrm{LH}$ and during continuous steady-state infusions of three graded doses of LH. The LH bolus disappearance curves provided estimates of metabolic clearance rates (MCR) of $24.1 \pm 4.7( \pm \mathrm{SD}) \mathrm{ml} / \mathrm{min}$ for bioactive $\mathrm{LH}$ vs. $56.2 \pm 12 \mathrm{ml} / \mathrm{min}$ for immunoactive $\mathrm{LH}$ in the same men $(P=0.03)$. A lower MCR of bioactive LH compared with immunoactive $\mathrm{LH}$ was also observed during continuous infusions of physiological doses of $\mathrm{LH}$; for example, the mean steady-state MCRs for bioactive and immunoactive $\mathbf{L H}$ were, respectively, $26.1 \pm 3.1$ and $34.2 \pm 3.2 \mathrm{ml} / \mathrm{min}(P=0.02)$. Moreover, the stepped-dose infusion regimens permitted us to demonstrate that increasing doses of pure human $\mathrm{LH}$ resulted in progressive and parallel decreases in the apparent MCRs of both bioactive and immunoactive $\mathrm{LH}$.

Based on the respective steady-state MCRs calculated at physiological plasma concentrations of immunoactive and bioactive $\mathrm{LH}$, we estimate a mean endogenous production rate for bioactive hormone of $1,937 \mathrm{IU} / 24 \mathrm{~h}$, and for immunoactive $\mathrm{LH}$ of $589 \mathrm{IU} / 24 \mathrm{~h}$ in normal men. These results indicate that previous estimates of $\mathrm{LH}$ production rates from immunoassay data alone markedly underestimate the quantity of biologically active hormone secreted in man.
\end{abstract}

\section{Introduction}

Reproductive function is maintained physiologically by the intermittent secretion of biologically active gonadotropic hormones. However, to date, the properties of luteinizing hormone $(\mathrm{LH})^{1}$ production and metabolic disposal have been understood almost exclusively in relation to immunoactive hormone (1-7). Although this approach provides important information regarding some aspects of gonadotropin dynamics, further studies have

Address correspondence to Dr. Veldhuis, Box 202, University of Virginia, School of Medicine, Charlottesville, VA 22908.

Received for publication 22 July 1985 and in revised form $18 \mathrm{No}$ vember 1985.

1. Abbreviations used in this paper: bioactive $\mathrm{LH}$, biologically active luteinizing hormone; HCG, human chorionic gonadotropin; HMG, human menopausal gonadotropin; IRP, International Reference Preparation; LH, luteinizing hormone; MCR, metabolic clearance rate; RICT, rat interstitial cell testosterone.

J. Clin. Invest.

(C) The American Society for Clinical Investigation, Inc.

0021-9738/86/04/1122/07 \$1.00

Volume 77, April 1986, 1122-1128 revealed significant discrepancies between immunoactive and bioactive LH release under conditions of either health or disease (8-14). In large part, these discrepancies reflect the inability of available immunoassays to directly measure the biologically active constituent(s) of LH in plasma.

In order to define the physiological properties of biologically active LH secretion and metabolic clearance, we have infused highly purified human LH in hypogonadotropic men. By employing both bolus injections and continuous equilibrium infusions of graded doses of $\mathrm{LH}$, we have been able to mimic a range of physiological LH concentrations under both dynamic and steady-state conditions. Moreover, in each circumstance, we have been able to directly compare the attributes of the plasma disposal of biologically active and immunoactive $\mathrm{LH}$ in the same subjects.

\section{Methods}

\section{Patient characteristics}

Six men with hypogonadotropic hypogonadism (immunoactive LH $<3 \mathrm{mIU} / \mathrm{ml}$, bioactive $\mathrm{LH}<1.6 \mathrm{mIU} / \mathrm{ml}$ ) participated in the study after provision of written informed consent, which was approved by the Human Investigation Committee of the University of Virginia School of Medicine (exogenous infusions), or the Clinica Medica V of the University of Rome (endogenous peaks). The patient characteristics are shown in Table I. Each individual had normal serum concentrations of cortisol, thyroxine, and prolactin, and normal hepatic, renal, and hematologic function. No patient had received any form of LH or human chorionic gonadotropin (HCG) for at least 2 mo before participation in the study.

\section{Clinical protocols}

$L H$ preparation(s). Two men were initially studied with a bolus injection of $35 \mu \mathrm{g}$ clinical grade human LH (National Institutes of Health, LH $82-45$ ). The bioactive potency of this preparation was only $2,225 \mathrm{IU} /$ $\mathrm{mg} \mathrm{LH}$ in the rat ventral prostate bioassay compared with an immunoreactive potency of 17,000 IU/mg LH (Second International Reference Preparation [IRP], of human menopausal gonadotropin [HMG]). The disappearance curves for this conventional but evidently impure LH preparation revealed markedly disproportionate clearance of immunoactive compared with biologically active LH (bioactive LH), with a nonphysiological increase in the bio/immuno LH ratio to a level of 8 12 (normal male range 3-5). Accordingly, this preparation was not used further in the present studies. Rather, we employed highly purified immunoassay grade human LH (NIADDK-hLH-I-2) prepared by Dr. A. F. Parlow with a potency of $9,500 \mathrm{IU} / \mathrm{mg} \mathrm{LH}$ by bioassay (ovarian ascorbic acid depletion), and $13,117 \mathrm{IU} / \mathrm{mg}$ by radioimmunoassay (RIA) (World Health Organization International Standard Urinary folliclestimulating hormone (FSH)/LH 70), associated with $8 \mathrm{IU} / \mathrm{mg}$ folliclestimulating hormone by RIA, and $0.03 \mathrm{IU} / \mathrm{mg}$ thyroid-stimulating hormone by RIA (World Health Organization human thyroid-stimulating hormone $68 / 38$ ). The potency of this preparation was $22,000 \mathrm{IU} / \mathrm{mg}$ by rat interstitial-cell testosterone bioassay (RICT) and $9,330 \mathrm{IU} / \mathrm{mg}$ by RIA (Second IRP; HMG standard).

Bolus injections. For the bolus injection studies, blood samples were 
Table I. Subject Characteristics

\begin{tabular}{llllll}
\hline Subject & Age & Height & Weight & Surface area & Diagnosis \\
\hline & $y r$ & $m$ & $k g$ & $m^{2}$ & \\
A & 22 & 1.72 & 74 & 1.90 & Kallmann's syndrome \\
B & 39 & 1.70 & 82 & 1.94 & Kallmann's syndrome \\
C & 33 & 1.78 & 78 & 1.93 & Kallmann's syndrome \\
D & 21 & 1.68 & 70 & 1.79 & $\begin{array}{c}\text { Hypopituitarism } \\
\text { (suprasellar tumor) }\end{array}$ \\
E & 29 & 1.78 & 75 & 1.90 & $\begin{array}{l}\text { Kallmann's syndrome } \\
\text { Fallmann's syndrome }\end{array}$ \\
\hline & 21 & 1.77 & 63 & 1.80 & Kalmann \\
\hline
\end{tabular}

withdrawn in the basal state at 15 -min intervals for $1 \mathrm{~h}$, after which 35 $\mu \mathrm{g}$ of this highly purified human LH was administered by bolus injection. Immediately after injection, blood samples were withdrawn serially at the following times: every $5 \mathrm{~min}$ for $30 \mathrm{~min}$; every $10 \mathrm{~min}$ for $\mathbf{4 0} \mathrm{min}$; every $15 \mathrm{~min}$ for $60 \mathrm{~min}$; and then, every $30 \mathrm{~min}$ for $180 \mathrm{~min}$.

The plasma from these samples was later assayed by RICT and RIA, and the corresponding disappearance curves analyzed by one or two compartment models (discussed further below).

Continuous equilibrium infusion protocols. Each of six men underwent stepped infusions comprising three doses of pure human $\mathrm{LH}$, which were divided into three phases as follows: Phase 1 began with basal sampling at 15-min intervals for $1 \mathrm{~h}$ followed by the bolus injection of $21 \mu \mathrm{g} \mathrm{LH}$ and the continuous infusion of $3.5 \mu \mathrm{g} \mathrm{LH} / \mathrm{h}$ for $4 \mathrm{~h}$ (four volunteers) or $6 \mathrm{~h}$ (two volunteers). The infusion was then stopped for $3 \mathrm{~h}$, until phase 2 was initiated by bolus injection of $34 \mu \mathrm{g} \mathrm{LH}$, followed by the continuous infusion of $14 \mu \mathrm{g} \mathrm{LH} / \mathrm{h}$ for 4-6 h. This infusion was then stopped for 3 $\mathrm{h}$, before initiating phase 3 , which comprised an $84-\mu \mathrm{g} \mathrm{LH}$ bolus injection followed by the continuous infusion of $35 \mu \mathrm{g} \mathrm{LH} / \mathrm{h}$ for 4-6 h. These infusion rates were chosen to approximate physiological concentrations of LH at lower doses and to test for saturability of clearance mechanisms at higher doses.

LH was dissolved in $0.25 \%$ human serum albumin/isotonic saline in a volume of $250 \mathrm{ml}$, which was sterilized by Millipore filtration (Waters Associates, Millipore Corp., Milford, MA). After bolus injections of a loading dose of $\mathrm{LH}$, continuous infusions were maintained by peristaltic pump at a rate of 40 or $60 \mathrm{ml} / \mathrm{h}$. Blood was sampled at 20-min intervals for the 4 or $6 \mathrm{~h}$ of each infusion phase to document attainment of equilibrium plasma LH concentrations. The exact quantities of $\mathrm{LH}$ infused were determined by bioassay and immunoassay of aliquots taken hourly from the actual infusion bottles.

Assays of $L H$. Plasma samples were assayed for immunoactive $\mathrm{LH}$ by double antibody RIA $(11,12)$, with a sensitivity of $1 \mathrm{mIU} / \mathrm{ml}$ in terms of the Second IRP of HMG, and for bioactive LH by the rat interstitial cell testosterone assay (RICT), with a sensitivity of $0.4 \mathrm{mIU} / \mathrm{ml}(8,12)$.

The RICT within-assay coefficient of variation averaged $8.5 \%$, with a range from $8.2 \%$ in normal men to $9.2 \%$ in children and postmenopausal women $(11,14)$. For the RIA, the coefficients of variation were $8.4 \%$ (at $2 \mathrm{mIU} / \mathrm{ml}$ ), $6.8 \%$ (at $11.5 \mathrm{mIU} / \mathrm{ml}$ ), and $4.6 \%$ (at $47 \mathrm{mIU} / \mathrm{ml}$ ) using $\mathrm{LH}$ antiserum that exhibits $\leq 15 \%$ crossreactivity with alpha subunit (11, 14). The LH contents of plasma and infusate samples from any single study session were all quantitated in the same assay.

Data analysis. The LH disappearance profiles were analyzed by assuming single or two-component exponential models, such that the instantaneous plasma LH concentration, [LH], at time $t$ was: [LH] $=\mathrm{A}_{0} \mathrm{e}^{-\alpha t}$ $+\mathrm{B}_{0} \mathrm{e}^{-\beta t}+\mathrm{C}$, where $(a) \mathrm{A}_{0}>0$ and $\mathrm{B}=0$ for a single-component fit; (b) $\mathrm{A}_{0}>0$ and $\mathrm{B}_{0}>0$ for a two-component model; (c) $\mathrm{C}$ represents the basal plasma LH concentration; and $(d) \alpha$ and $\beta$ are rate constants. The parameters were estimated by Gauss-Newton iteration with confidence limits for the precision of fit (15).
The MCR was calculated from the equation:

$\mathrm{MCR}=\frac{\alpha \times \beta}{\mathrm{A}_{0} \beta+\mathrm{B}_{0} \alpha} \times D$

where $D$ is the total dose of LH administered.

Under steady-state infusion conditions, the MCR was calculated as: $\mathrm{MCR}=$ infusion rate/[LH $]_{\text {mean }}$, where $[\mathrm{LH}]_{\text {mean }}$ represents the mean steady-state LH concentration attained during the infusion. The infusion rate is the measured rate of $\mathrm{LH}$ infusion $(\mathrm{mIU} / \mathrm{min}$ ) by assay of hourly aliquots of infusate.

The relationship between MCR and plasma LH concentration ([LH]) was assessed by nonlinear, least-squares curve fitting of the data to the function:

$\mathrm{MCR}=\frac{\mathrm{MCR}_{0}}{1+\alpha[\mathrm{LH}]}+\mathrm{MCR}_{\mathrm{inf}}$,

where $M \mathrm{CR}_{0}$ represents the $M C R$ increment above $\mathbf{M C R}_{\text {inf }}$ when $M C R$ is extrapolated to a zero plasma LH concentration, $\alpha$ is a slope parameter, and $\mathbf{M C R}_{\text {inf }}$ is the MCR extrapolated toward infinite [LH]. This function was used simply to insure that an inverse, nonlinear relationship between [LH] and MCR could be estimated if present. A relationship that approximated linearity would be defined by a slope factor $(\alpha)$ that approached zero (15).

Comparisons between bioactive and immunoactive $\mathrm{LH}$ values were analyzed by within-subject comparisons using Student's paired two-tailed $t$ test or the Mann-Whitney test in the case of bio/immuno ratios (16). Analysis of variance with correction for repeated measures was used to assess changes in relation to the three stages of the infusions (16). Significance was assumed for $P<0.05$.

\section{Results}

Bolus injection of pure human LH. Four men (subjects A-D in Table I) were sampled basally at 15-min intervals for $1 \mathrm{~h}$, and then administered $35 \mu \mathrm{g}$ of pure human $\mathrm{LH}$ intravenously as a bolus. The subsequent simultaneous disappearance curves for bioactive and immunoactive $\mathrm{LH}$ demonstrated a rapid rise in plasma LH concentrations immediately after bolus injection (Fig. 1). Peak measured plasma LH concentrations were significantly higher for bioactive $\mathrm{LH}(275 \pm 19 \mathrm{mIU} / \mathrm{ml})$ than immunoactive LH $(72 \pm 5 \mathrm{mIU} / \mathrm{ml})(P<0.01)$. However, in both cases, $\mathrm{LH}$ concentrations exhibited an initially rapid decline over 1-2 $\mathrm{h}$ followed by more delayed disappearance over 3-6 h.

As further illustrated in the lower panel of Fig. 1, the plasma bio/immuno $\mathrm{LH}$ ratio increased from a mean of $1.2 \pm 0.4$ (median 0.9 ) basally to a mean of $3.8 \pm 0.2$ (median 3.8 ) within $5 \mathrm{~min}$ of bolus $\mathrm{LH}$ injection $(P<0.001)$. The bio/immuno $\mathrm{LH}$ ratio then gradually increased further to a peak mean value of $5.6 \pm 0.4$ (median 5.3) at 60 min after the bolus administration of LH ( $P$ $<0.05$ vs. 5 -min value). This value is similar to that reported for endogenous $\mathrm{LH}$ peaks $(5.27 \pm 1.60)(12)$. During the remaining portion of the disappearance curves, the bio/immuno ratio was stable at a mean value of $4.64 \pm 0.3$ (median 4.1 ), which is not significantly different from the mean value observed in normal men between pulses (12).

The disappearance curves for plasma concentrations of bioactive and immunoactive LH were analyzed by one- and two-component exponential equations. When the disappearance curves were analyzed by single-component exponential decay, the calculated peak amplitudes $\mathrm{A}_{\mathbf{0}}$ for plasma concentrations of bioactive and immunoactive LH differed significantly (340 194 vs. $66 \pm 6)(P=0.05)$, as did the alpha (rate-constant) components 

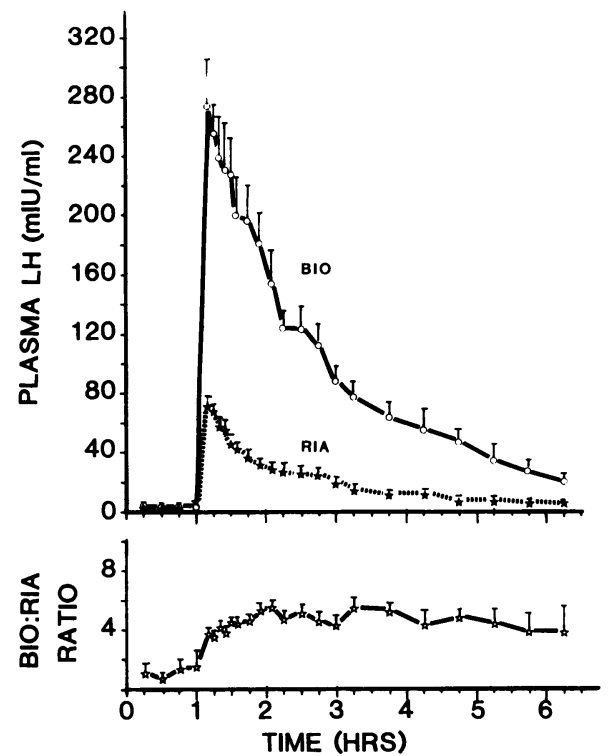

Figure 1. Simultaneous plasma disappearance profiles for bioactive (BIO) and immunoactive (RIA) LH after bolus intravenous injection of $35 \mu \mathrm{g}$ of highly purified LH. The lower panel depicts the plasma bio/immuno LH ratios. Data are means \pm SEM for four hypogonadotropic men.

which corresponded to estimated half-lives of disappearance of $65.3 \pm 4.9 \mathrm{~min}$ for bioactive $\mathrm{LH}$ vs. $46.7 \pm 6.8 \mathrm{~min}$ for immunoactive LH $(P<0.01)$. When analyzed by two-component fitting (summarized in Table II), the calculated amplitudes of both the large and small exponential components were considerably greater for bioactive LH than for immunoactive LH ( $P$ $=0.003$ for larger component). Comparison of the corresponding apparent half-lives of disappearance of bioactive and immunoactive $\mathrm{LH}$ also revealed more rapid immunoactive $\mathrm{LH}$ disappearance for the rapid component, although this difference did not attain statistical significance.

The preceding analyses indicated that the disappearance properties for human LH differed between bioactive and immunoactive hormones consistently in the amplitude of the more slowly disappearing component, which averaged sixfold larger for bioactive than immunoactive LH. This difference was considerably greater than could be accounted for by the bio/immuno ratio in the infusate (mean $2.43 \pm 0.17$ [ $N=108$ samples]). Thus, we tested whether the initial volumes of distribution of bioactive and immunoactive $\mathrm{LH}$ were significantly different. As shown in Table III $A$, independently of the modeling of the disappearance curves, the distribution volumes for immunoactive $\mathrm{LH}$ were significantly greater than those for bioactive hormone in the same subjects $(P<0.001)$.

Based upon the disappearance properties of bioactive and immunoactive $\mathrm{LH}$, the apparent metabolic clearance rates (MCRs) for these two species could be calculated (Table III $B$ ). The mean MCR for bioactive $\mathrm{LH}$ was $24.1 \pm 2.4$ ( \pm SEM) $\mathrm{ml} /$ min compared with $56.4 \pm 6 \mathrm{ml} / \mathrm{min}$ for immunoactive LH ( $P$ $<0.03$ ) in the two-compartment model. These values were not significantly different from the corresponding estimates for a single-compartment model. Accordingly, after bolus injection of LH, the apparent MCR for bioactive hormone is less rapid than that for immunoactive hormone in the same individuals, independently of the disappearance model chosen. Nonetheless, the absolute values of the apparent MCR for bioactive and immunoactive LH were highly correlated $(R=+0.977, P<0.01)$.

Continuous graded infusions of $L H$. In six subjects $(A-F$, Table I), pure human LH was administered by bolus injection followed by continuous infusion over three dose ranges. This steady-state technique serves to eliminate possible influences of discrepant distribution volumes. Table IV summarizes the mean plasma concentrations of bioactive and immunoactive LH attained during each of the three phases of LH infusion. Four men were infused for $4 \mathrm{~h}$ and two men for $6 \mathrm{~h}$, with blood sampled at 20-min intervals during the infusions to obtain accurate estimates of mean steady state plasma $\mathrm{LH}$ concentrations. The results in the four men infused over $4 \mathrm{~h}$ are summarized in Fig. 2 , which clearly indicate the attainment of steady-state plasma concentrations of both bioactive and immunoactive LH during this interval. Note that the mean plasma concentrations of bioactive and immunoactive $\mathrm{LH}$ attained during the low-dose infusions (Phase I) were within the physiological range of LH measurements for normal healthy adults $(11,12,14)$. The higher infusion doses were used to examine the relationship between MCR and plasma LH concentration and test for saturation of metabolic clearance processes (as discussed further below).

Fig. 3 depicts the individual MCRs for immunoactive and bioactive $\mathrm{LH}$ in each subject studied in relationship to the three doses of LH infused continuously (Phases 1-3). For Phase 1 (low-dose) infusion, the mean MCRs for bioactive and immunoactive $\mathrm{LH}$ were, respectively, $28.3 \pm 3.4$ and $37.1 \pm 3.5 \mathrm{ml} / \mathrm{min}$ $(P<0.02)$. Notably, at higher doses of infusion, the apparent

Table II. Disappearance Curves for Highly Purified Human LH after Bolus Injection

\begin{tabular}{|c|c|c|c|c|c|c|c|}
\hline \multirow[b]{2}{*}{ Subject } & \multicolumn{2}{|c|}{ Two-component fitting parameters* } & \multirow[b]{2}{*}{$\alpha \times 10^{-2}$} & \multirow[b]{2}{*}{$\beta \times 10^{-3}$} & \multirow{2}{*}{$\frac{t_{1 / 2}}{\alpha}$} & \multirow{2}{*}{$\frac{t_{1 / 2}}{\beta}$} & \multirow[b]{2}{*}{$\mathbf{C}$} \\
\hline & $A_{0}$ & $\mathbf{B}_{0}$ & & & & & \\
\hline & & & & & $\min$ & $\min$ & \\
\hline Bio LH & $\begin{array}{l}95 \\
(54-125)\end{array}$ & $\begin{array}{l}211 \dagger \\
(177-256)\end{array}$ & $\begin{array}{l}2.18 \\
(1.47-2.95)\end{array}$ & $\begin{array}{l}8.14 \\
(7.57-8.89)\end{array}$ & $\begin{array}{l}31.7 \\
(23-47)\end{array}$ & $\begin{array}{l}85.2 \\
(78-92)\end{array}$ & $2.5 \pm 1$ \\
\hline RIA LH & $\begin{array}{l}56 \\
(40-72)\end{array}$ & $\begin{array}{l}35 \\
(25-47)\end{array}$ & $\begin{array}{l}3.87 \\
(2.67-5.24)\end{array}$ & $\begin{array}{l}7.69 \\
(6.27-9.32)\end{array}$ & $\begin{array}{l}17.9 \\
(13-26)\end{array}$ & $\begin{array}{l}90.1 \\
(74-111)\end{array}$ & $3.4 \pm 1$ \\
\hline
\end{tabular}

* Plasma LH concentrations were fit to the bi-exponential equation: $[\mathrm{LH}]=\mathrm{A}_{0} \mathrm{e}^{-\alpha t}+\mathrm{B}_{0} \mathrm{e}^{-\beta t}+\mathrm{C} . \quad \ddagger P=0.003$ for bioactive (Bio) vs. immunoactive (RIA) LH. Parentheses give $67 \%$ confidence limits for the asymmetric variance spaces for the four individuals studied. 
Table III.

A. Apparent Distribution Volumes of Bioactive and Immunoactive $\mathrm{LH}$ after Bolus Injection

\begin{tabular}{llllll}
\hline & \multicolumn{2}{l}{ Vo* Bioactive LH } & & Vo RIA LH & \\
\cline { 2 - 3 } \cline { 5 - 6 } Subject & 1-component & 2-component & & 1-component & 2-component \\
\hline Means \pm SEM & $2.21 \pm 0.29^{\mathrm{a}}$ & $2.15 \pm 0.28^{\mathrm{a}}$ & & $3.82 \pm 0.35^{\mathrm{b}}$ & $3.47 \pm 0.31^{\mathrm{b}}$
\end{tabular}

Disappearance curves were fit to either a 1- or a 2-component model, as indicated. * Vo, apparent volume of distribution (liters). Differing superscripts are significantly different at $P<0.01$.

B. Metabolic Clearance Rates of Biologically Active and Immunologically Active Human LH Calculated after Bolus Injection

from 1- or 2-Component Model of Disappearance

\begin{tabular}{llllll}
\hline & \multicolumn{2}{l}{ MCR Bioactive LH } & & \multicolumn{2}{l}{ MCR Immunoactive LH } \\
\cline { 2 - 3 } \cline { 5 - 6 } & 1-component & 2 2-component & & 1 -component & 2-component \\
\hline & $\mathrm{ml} / \mathrm{min}$ & $\mathrm{ml} / \mathrm{min}$ & & $\mathrm{ml} / \mathrm{min}$ & $\mathrm{ml} / \mathrm{min}$ \\
Means \pm SEM & $24.3 \pm 2.8^{*}$ & $24.1 \pm 2.4^{*}$ & & $59.4 \pm 6$ & $56.4 \pm 6$
\end{tabular}

$35 \mu \mathrm{g}$ of highly purified human $\mathrm{LH}$ were injected intravenously as a bolus in four hypogonadotropic men. The subsequent bioactive and immunoactive LH disappearance curves were analyzed for 1- or 2component exponential decay. The metabolic clearance rates of bioactive and immunoactive $\mathrm{LH}$ were then calculated from the integrated areas under the 1- or 2-component disappearance curves (Methods). * $P=0.03$ for bioactive vs. immunoactive MCRs.

MCRs for bioactive and immunoactive LH both declined progressively. Comparative MCRs for bioactive and immunoactive LH, respectively, at Phase II were $18.6 \pm 3.6$ vs. $31.6 \pm 4.0 \mathrm{ml} /$ $\min (P<0.01)$, and at Phase III, were $9.3 \pm 1.0$ vs. $16.4 \pm 2.3 \mathrm{ml} /$ $\min (P<0.01)$.

The apparent inverse relationship between plasma LH concentration and MCR across these three dose-ranges was evaluated for each of the 17 infusion sets undertaken. (One Phase I infusion was not analyzed because of problems with the intravenous catheter). Higher plasma concentrations of LH, whether assessed by immunoassay or bioassay, were associated with a progressive

Table IV. Constant Infusion of Highly Purified Human LH

\begin{tabular}{lccll}
\hline & Plasma Bio LH & Plasma RIA LH & Plasma B:I ratio & $\begin{array}{c}\text { Infusate } \\
\text { B:I ratio }\end{array}$ \\
\hline & $m I U / m l$ & & & \\
Basal & $2.0 \pm 1.8$ & $4.7 \pm 1.2$ & $0-1.6^{*}$ & \\
Phase I & $66.4 \pm 15.2 \ddagger$ & $21.2 \pm 2.8$ & $2.90 \pm 0.11$ & $2.3 \pm 0.3$ \\
Phase II & $253.6 \pm 54.9 \ddagger$ & $65.0 \pm 13.0$ & $3.73 \pm 0.20$ & $2.5 \pm 0.4$ \\
Phase III & $711.5 \pm 121 \ddagger$ & $182.5 \pm 28.9$ & $3.69 \pm 0.22$ & $2.5 \pm 0.3$
\end{tabular}

Data are means $\pm \mathrm{SD}$ ( $n=6$ subjects, each sampled at 20-min intervals during the continuous infusion except in Phase $\mathrm{I}$, where $n=5$, since one of the low-dose infusions was technically flawed).

* Range (5 of 6 B:I ratios were zero).

$\ddagger P<0.01$ vs. RIA.

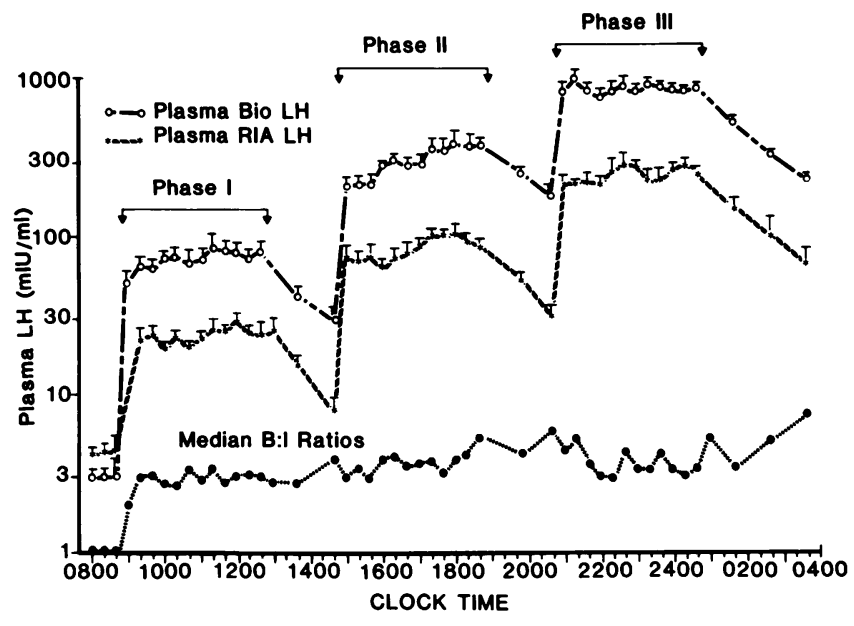

Figure 2. Simultaneous plasma concentrations of bioactive (BIO) LH and immunoactive (RIA) LH during three-phase continuous infusions of highly purified human LH. The plasma concentrations of bioactive and immunoactive LH (upper and middle curves) were measured at the indicated intervals in response to three stepped infusions of $\mathrm{LH}$, denoted as Phase I, II, or III (Methods). The median plasma bio/immuno (B/I) LH ratios are depicted in the lower curve. Other data are means ( $\pm \mathrm{SEM}, N=4$ men).

decline in the apparent MCR (Fig. $4 A$ [bioactive LH] and Fig. $4 B$ [immunoactive $L H]$ ). Moreover, at the highest plasma concentrations of LH attained, there was a clear tendency for the MCR to plateau toward a nadir value. Based on non-linear leastsquares curve-fitting, the apparent plateau MCR for bioactive LH was $8.4 \pm 0.77 \mathrm{ml} / \mathrm{min}$ and for immunoactive LH $13.5 \pm 2.42$ $\mathrm{ml} / \mathrm{min}$. As discussed further below, this relationship between plasma LH concentration and corresponding MCR for bioactive and immunoactive hormone permitted us to calculate production rates at plasma $\mathrm{LH}$ concentrations that approximated physiological levels.

During the three graded-dose infusions of LH, mean steady state plasma concentrations of bioactive and immunoactive $\mathrm{LH}$ attained were highly correlated $(R=+0.917, P<0.01)$, but with consistently higher plasma concentrations of bioactive compared with immunoactive $\mathrm{LH}(P<0.001)$. This relationship was approximated by a linear equation with a slope of 3.71 , indicating that the bio/immuno $\mathrm{LH}$ ratio in plasma across a

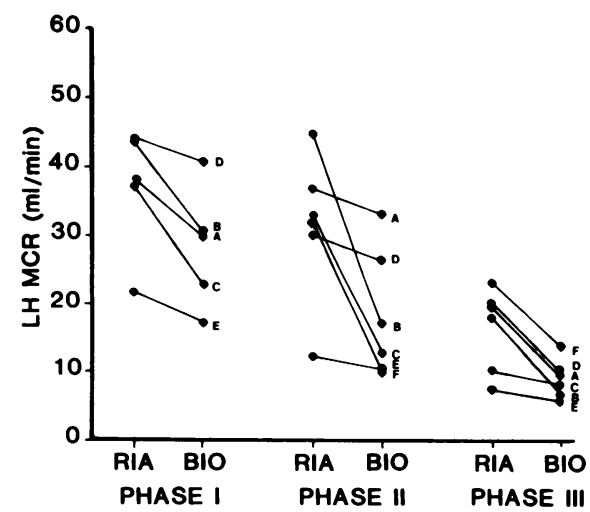

Figure 3. Individual MCRs for immunoactive (RIA) or bioactive (BIO) LH during each of the three phases of LH infusions shown. The letters correspond to subjects listed in Table I. 

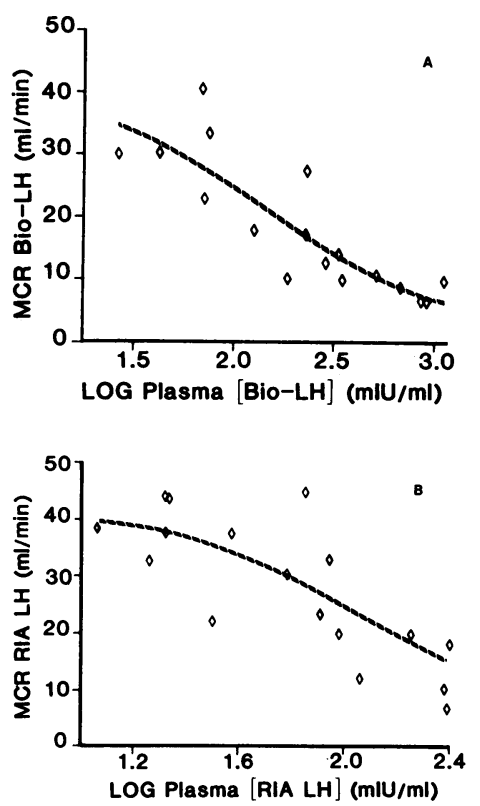

Figure 4. Relationship between the MCR of bioactive (BIO) LH and the plasma concentration of BIO-LH $(A)$; and between the MCR of immunoactive (RIA) LH and its plasma concentration $(B)$ in 17 individual equilibrium infusion sessions.

wide range of infusions approximated 3.71. This value is similar to that measured either in normal men (3.26 during the interpulse interval, and 3.95 overall), or during the continuous infusions (mean 3.44), but is consistently higher than the bio/immuno LH ratio in the injected preparation (2.36) or the infusate proper (average 2.43). The higher bio/immuno LH ratio in plasma compared with infusate presumably reflected the lesser clearance of bioactive LH compared with immunoactive LH during all phases of the infusion. Accordingly, the estimated MCR for bioactive LH was uniformly lower than that for immunoactive LH in these 17 infusion sets. Across the wide range of infusion rates tested, the MCR for bioactive LH averaged $66.3 \%$ of that for immunoactive $\mathrm{LH}$ in the same individual $(P<0.001$, where $R=+0.767$ ) (Fig. 5). Thus, we infer that the increase in plasma bio/immuno $\mathrm{LH}$ ratios in men infused with pure human $\mathrm{LH}$ occurs in part because of relatively reduced metabolic clearance of bioactive compared with immunoactive hormone.

The production rate of $\mathrm{LH}$ was calculated from mean MCRs for bioactive and immunoactive $\mathrm{LH}$ achieved at physiological plasma hormone concentrations. Assuming a mean physiological plasma bioactive $\mathrm{LH}$ concentration of $41.4 \mathrm{mIU} / \mathrm{ml}$ in normal men $(11,12)$, we calculated a corresponding mean MCR for bioactive $\mathrm{LH}$ of $32.5 \mathrm{ml} / \mathrm{min}$ from the best fit of the relationship between plasma bioactive $\mathrm{LH}$ concentration and MCR for bioactive LH (Fig. $4 A$ ). Correspondingly, the mean plasma immunoactive LH concentration for normal men in our assay is $10.2 \mathrm{mIU} / \mathrm{ml}(11,12)$, to which would be associated a mean

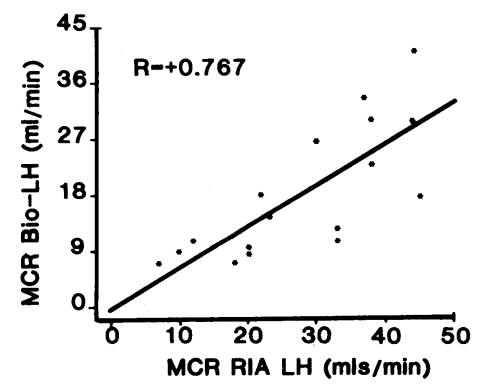

Figure 5. Correlation between the MCRs of bioactive and immunoactive $\mathrm{LH}$. Data are values from 17 individual equilibrium infusion sessions.
MCR of $40.1 \mathrm{ml} / \mathrm{min}$. Based upon these values, we estimate a mean production rate for bioactive $\mathrm{LH}$ of $1,346 \mathrm{mIU} / \mathrm{min}(1,937$ $\mathrm{IU} / 24 \mathrm{~h}$ ). This compares with a mean production rate for immunoactive $\mathrm{LH}$ in the same individuals of $409 \mathrm{mIU} / \mathrm{min}$ (589 IU/24 h).

At the physiological plasma concentrations of bioactive and immunoactive LH used above, the ratio of the MCRs for bioactive/immunoactive hormone was 0.77 . In contrast, estimated production rates differed by 3.3 -fold. Since the ratio of plasma bioactive to immunoactive $\mathrm{LH}$ concentrations is 3.95 under physiological conditions, we infer that the 3.3-fold difference in production rate (rather than the slightly lower MCR) of bioactive compared with immunoactive $\mathrm{LH}$ would best account for the higher plasma bio/immuno LH ratio of 3.95 in normal men.

Endogenous $\mathrm{LH}$ pulses. To evaluate endogenous $\mathrm{LH}$ disappearance rates, blood was withdrawn at 4-10-min intervals for 2-3 h, in six healthy men. The half-lives of disappearance were calculated for endogenous bioactive LH peaks, if at least four consecutively decreasing LH concentrations were measured (Fig. 6). This permitted the identification of 15 endogenous bioactive $\mathrm{LH}$ peaks, with a mean single-component half-life of $13.9 \pm 4.4(\mathrm{SD}) \mathrm{min}$ and a range of $5.5-43 \mathrm{~min}$. Although this estimate may include some contribution of initial distribution/ equilibration as well as possibly continued secretion, the values encompass the range (5-48 min) we observed for exogenously injected, highly purified human LH in hypogonadotropic men.

\section{Discussion}

Available studies of the metabolic clearance of human $\mathrm{LH}$ from plasma have emphasized that calculated clearance rates, although validated in several ways, provide estimates of only immunoactive $\mathrm{LH}$, rather than biologically active hormone (1-7). This distinction is particularly important, because recent investigations have disclosed significant discordance between measurements of immunoactive and biologically active $\mathrm{LH}$ under various pathophysiological conditions (8-14). Significant disparities have

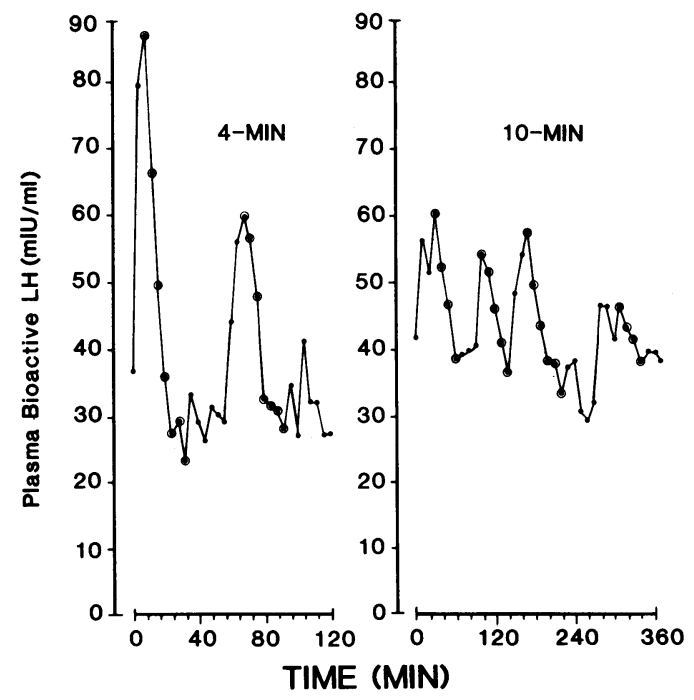

Figure 6. Disappearance properties of endogenous bioactive LH assessed in spontaneously occurring peaks. Normal men underwent blood withdrawal at 4-min or 10-min intervals for 2 or $3 \mathrm{~h}$, as illustrated for two individuals. The particular points used to estimate endogenous bioactive LH disposal in these subjects are circled. 
also been reported between bioassay and immunoassay (RIA) estimates for HCG (17). Accordingly, in the present study, we have used a sensitive and precise bioassay (RICT) system to delineate physiological properties of the plasma disposal of bioactive $\mathrm{LH}$. These studies have been performed with the most highly purified form of human $\mathrm{LH}$ available to date (approximately 3-8-fold more active than earlier preparations). Moreover, by using both RIA and RICT, we have been able to compare directly the metabolic clearance of immunoactive and bioassayable LH activity in the same individuals. These comparisons have been validated by investigating LH clearance under nonequilibrium conditions (bolus injection), as well as during steadystate infusions of various doses of $\mathrm{LH}$ designed to encompass physiological plasma LH concentrations.

Comparison of the clearance estimates for immunoactive and bioassayable LH activity under nonequilibrium conditions revealed significant differences. For example, whether disappearance curves were assumed to conform to a one- to twocomponent model of exponential decay, the magnitude of the peak plasma bioactive $\mathrm{LH}$ concentration was consistently greater than that for immunoactive LH. This disparity could be accounted for in part by discrepant initial distribution volumes, since those for immunoactive $\mathrm{LH}$ were 1.6-1.7-fold larger than for bioactive LH. Analogous differences between bioactive and immunoactive plasma distribution volumes have been reported for HCG in three amenorrheic women given bolus HCG injections (17). Although the exact basis for the apparently larger distribution volumes associated with immunoactive LH or HCG compared with their bioactive counterparts is not known, we speculate that carbohydrate-poor constituents of the glycoprotein preparations that are less bioactive but retain immunoactivity are removed more extensively by specific hepatic, renal, and/or reticuloendothelial receptors, which tend to accumulate carbohydrate-impoverished proteins more efficiently (18). This hypothesis is consistent with our initial results with the relatively impure LH preparation in which LH immunoactivity was removed very rapidly with resulting increases in bio/immuno $\mathbf{~} H$ ratios. Ultimately, this consideration would need to be tested in the human by the injection of synthetically pure LH glycoproteins with varying specific carbohydrate content.

Our results indicated a significantly lower MCR for bioactive vs. immunoassayable LH activity after bolus injection. This difference was associated with a tendency for LH measured by bioassay to exhibit a slower plasma disappearance rate than $\mathrm{LH}$ measured by immunoassay. The discrepant disappearance rates for bioactive vs. immunoactive estimates of LH activity after bolus injection might be attributable to distinct subunit and/or carbohydrate compositions of LH detected in the two assays, since the subunit composition and sugar content of certain glycoprotein hormones, including LH and HCG, influence MCRs (17-21). Alternatively, the highly purified nature of the present LH preparation may have altered its biological composition significantly compared with that recognized in normal plasma. This does not seem to be the case, because: $(a)$ bolus exogenous LH injections resulted in plasma bio/immuno LH ratios and plasma bioactive LH disappearance rates very similar to those observed in spontaneous endogenous LH peaks; $(b)$ continuous infusions of exogenous $\mathrm{LH}$ resulted in plasma bio/immuno LH ratios very similar to those observed in endogenous interpulse basal intervals in normal men; and $(c)$ the immunoactive LH disappearance rates for this exogenous preparation closely matched those ob- served for endogenous LH after surgical or medical hypophysectomy (13-26 min in the present study, and 17.5-26 min after surgical hypophysectomy [2] or 8-27 min after medical hypophysectomy using a selective gonadotropin-releasing hormone antagonist) [Veldhuis, J. D., M. L. Davis, A. D. Rogol, M. L. Dufau, and K. J. Catt, unpublished observations]).

The preceding MCRs for bioactive and immunoassayable LH activity were derived from non-steady-state experiments, which were associated with discordant initial plasma distribution volumes, disappearance rates, and possibly varying degrees of interconversion of $\mathrm{LH}$ activity and/or saturation of clearance mechanisms for immunoactive and bioactive hormone. To address these considerations, we studied six individuals during continuous equilibrium infusions. Such steady-state conditions remove the influence of variable plasma distribution volumes, hormonal interconversion, and nonuniform initial disappearance rates on estimates of MCR. Moreover, a paradigm of three graded doses of human LH that encompassed a broad range of plasma LH concentrations allowed us to examine MCR's at physiological levels of LH, and to test for concentration-dependence and saturability of clearance mechanisms.

Our results from 17 individual equilibrium infusion sessions embracing a range of plasma $\mathrm{LH}$ concentrations document a significant inverse relationship between plasma $\mathrm{LH}$ levels and apparent MCR. In the case of both immunoactive and bioassayable LH activity, higher rates of LH infusion were associated with progressive decreases in MCR. The highest doses of LH tested resulted in MCRs for bioactive and immunoactive $\mathrm{LH}$ that approached plateau values, suggesting that LH clearance mechanisms are saturable at high plasma hormone concentrations. This relationship may account in part for the range of immunoactive LH MCRs reported to date in response to widely differing infusion rates, and may also explicate some of the disparity observed between bioactive and immunoactive LH MCRs after bolus injection. Notably, after bolus injection, simultaneous plasma concentrations of the two species differ significantly, and consequently occupy different regions on the respective curves relating plasma concentrations to MCR.

The distinctive relationships between plasma LH concentration and MCR applicable to bioactive and immunoassayable LH activity permitted us to more accurately approximate the average MCR at LH concentrations that are attained physiologically in man. We estimated that in normal men the mean MCR for bioactive $\mathrm{LH}$ would be $32.5 \mathrm{ml} / \mathrm{min}$ (at a mean plasma bioactive LH concentration of $41.4 \mathrm{mIU} / \mathrm{ml}$ ) and for immunoactive $\mathrm{LH} 40.1 \mathrm{ml} / \mathrm{min}$ (at a corresponding plasma immunoactive $\mathrm{LH}$ concentration of $10.2 \mathrm{mIU} / \mathrm{ml}$ ). These data permitted us to estimate mean production rates of bioactive and immunoactive $\mathrm{LH}$ in normal men as $1,937 \mathrm{IU} / 24 \mathrm{~h}$ and 589 IU/24 h, respectively. Notably, the apparent production rate for LH bioactivity is approximately 3.3-fold higher than that for LH immunoactivity. In contrast, the MCR for LH bioactivity was only $33 \%$ less than that for immunoactivity. Accordingly, we infer that the markedly higher production rate of bioactive compared to immunoassayable $\mathrm{LH}$ activity is the predominant factor accounting for the high plasma bio/immuno $\mathrm{LH}$ ratio characteristic of normal men under physiological conditions. ${ }^{2}$

2. This inference is based upon the algebraic relationship between production rate and MCR under steady-state conditions (production rate $=$ MCR $\times$ [hormone]). The calculation of production rate assumes a 
The present studies using the RICT bioassay indicate that previous estimates of $\mathrm{LH}$ production rates from immunoassay data alone markedly underestimate the quantity of biologically assayable hormone secreted under physiological conditions. In contrast, our measurements of immunoactive LH clearance in the same subjects are similar to those reported by other investigators using unlabeled hormone, or immuno-precipitation of radiolabeled LH (1-7). For example, our mean MCR for immunoactive $\mathrm{LH}$ of $34.2 \pm 3.2 \mathrm{ml} / \mathrm{min}$ in men coincides with that of Marshall et al. (21.3 $\pm 2.6 \mathrm{ml} / \mathrm{min}$ [3]), and that of Pepperell et al. (43.5 $\pm 8.2 \mathrm{ml} / \mathrm{min}[6])$. Thus, our contrasting results with the RICT bioassay suggest that immunospecific methods significantly underestimate the quantities of bioassayable $\mathrm{LH}$ activity produced in normal man. In addition, various investigators have reported that sensitive and specific bioassays and radioimmunoassays for LH typically detect bio/immuno $\mathrm{LH}$ ratios in excess of unity in normal male plasma $(8,10-12)$. This suggests that many available $\mathrm{LH}$ immunoassays fail to recognize the majority of biologically assayable hormone present in the circulation. Similar discrepancies also appear in the identification of bioactive and immunoactive LH pulses in normal men, healthy young women, and postmenopausal individuals, since approximately $16 \%$ of bioactive $\mathrm{LH}$ pulses are detected without any significant increase in blood immunoactive LH concentrations $(11,12,14)$.

In summary, we have utilized a highly specific, sensitive and precise bioassay of $\mathrm{LH}$ to appraise the metabolic clearance of LH bioactivity in man. We investigated the plasma disposal of highly purified LH using both nonequilibrium conditions as well as a range of steady-state infusions. These combined approaches allowed comparisons of metabolic clearance rates for both immunoactive and bioassayable $\mathrm{LH}$ activity in the same individuals. Our results document certain pitfalls in the analysis of immunoactive LH disappearance after bolus injection, while offering quantitative estimates of the metabolic clearance and production rates of biologically active $\mathrm{LH}$. We believe these results provide an important basis for further investigations of the plasma disposal of LH bioactivity. Moreover, these also serve to underscore the restricted ability of immunoassay estimates to reflect fully the behavior of bioactive $\mathrm{LH}$ in the circulation.

\section{Acknowledgments}

We thank Chris McNett for her skillful preparation of the manuscript; Paula P. Azimi for the artwork; the National Hormone and Pituitary Program for the provision of purified human LH; Sandra Jackson and the expert nursing staff at the Clinical Research Center at the University of Virginia for conduct of the research protocols; and Dr. Michael L. Johnson for generous provision of his nonlinear curve-fitting programs.

This work was supported in part by National Institutes of Health Biomedical Research Support Award 5S07RR05431; University of Virginia Computer Services Grant, RCDA 1 K04 HD00634 and NIDA Grant R03DA03315 (to Dr. Veldhuis); U. S. Public Health Service General Clinical Research Grant RR-847; and Diabetes Research and Training Center Grant 5-P60-AM-22125-05.

relationship between the infused and circulating hormone species (see Discussion). Subject to this reasonable assumed relationship, the normal plasma bio/immuno LH ratio of $\sim 3.95$ in men would result predominantly from a 3.3-fold increase in bioactive $\mathrm{LH}$ production rate, and to a lesser degree, from a slight (33\%) decrease in bioactive LH clearance.

\section{References}

1. Kohler, P. O., G. T. Ross, and W. D. Odell. 1968. Metabolic clearance and production rates of human luteinizing hormone in preand post-menopausal women. J. Clin. Invest. 47:38-44.

2. Yen, S. S. C., O. Llerena, B. Little, and O. H. Pearson. 1968. Disappearance rates of endogenous luteinizing hormone and chorionic gonadotropin in man. J. Clin. Endocrinol. Metab. 28:1763-1768.

3. Marshall, J. C., D. C. Anderson, T. R. Frazer, and P. Harsoulis. 1973. Human luteinizing hormone in man: studies of metabolism and biological action. J. Endocrinol. 56:431-439.

4. Schalch, D. S., A. F. Parlow, R. C. Boon, and S. Reichlin. 1968. Measurement of human luteinizing hormone in plasma by radioimmunoassay. J. Clin. Invest. 47:665-678.

5. Raiti, S., T. P. Foley, R. Penny, and R. M. Blizzard. 1975. Measurement of the production rate of human luteinizing hormone using the urinary excretion technique. Metab. Clin. Exp. 24:937-941.

6. Pepperell, R. J., D. M. de Kretser, and H. G. Burger. 1975. Studies on the metabolic clearance rate and production rate of human luteinizing hormone and on the initial half-time of its subunits in man. J. Clin. Invest. 56:118-126.

7. Wehmann, R. E., M. R. Blackman, and S. M. Harman. 1982. Metabolic clearance rates of luteinizing hormone in women during different phases of the menstrual cycle and while taking an oral contraceptive. J. Clin. Endocrinol. Metab. 55:654-659.

8. Dufau, M. L., R. Pock, A. Neubauer, and K. J. Catt. 1976. In vitro bioassay of $\mathrm{LH}$ in human serum: the rat interstitial cell testosterone (RICT) assay. J. Clin. Endocrinol. Metab. 42:958-969.

9. Dufau, M. L., I. Beitins, J. McArthur, and K. J. Catt. 1977. Bioassay of serum LH concentration in normal and LHRH stimulated human subjects. In The Testis in Normal and Infertile Men. P. Troen and H. R. Nakin, editors. Raven Press, New York.

10. Robertson, D. M., V. Puri, M. Lindberg, and E. Diczfalusy. 1979. Biologically active luteinizing hormone (LH) in plasma. V. A re-analysis of the differences in the ratio of biological to immunological $\mathrm{LH}$ activities during the menstrual cycle. Acta Endocrinol. 92:615-626.

11. Dufau, M. L., J. D. Veldhuis, F. Fraioli, M. L. Johnson, and I. Z. Beitins. 1983. Mode of bioactive luteinizing hormone secretion in man. J. Clin. Endocrinol. Metab. 57:993-1000.

12. Veldhuis, J. D., A. D. Rogol, M. L. Johnson, and M. L. Dufau. 1983. Endogenous opiates modulate the pulsatile secretion of biologically active luteinizing hormone in man. J. Clin. Invest. 72:2031-2040.

13. Sawyer-Steffan, J. E., B. L. Lasley, J. D. Hoff, and S. S. C. Yen. 1982. Comparison of in-vitro bioactivity and immunoreactivity of serum LH in normal cyclic and hypogonadal women treated with low doses of LH-RH. J. Reprod. Fertil. 65:45-51.

14. Veldhuis, J. D., I. Z. Beitins, M. L. Johnson, M. A. Serabian, and M. L. Dufau. 1984. Biologically active luteinizing hormone is secreted in episodic pulsations that vary in relation to stage of the menstrual cycle. J. Clin. Endocrinol. Metab. 58:1050-1058.

15. Johnson, M. L. 1983. Evaluation and propagation of confidence intervals in nonlinear, asymmetrical variance spaces. Biophys. J. 44: 101-106.

16. Winer, B. J. 1971. Statistical Principles in Experimental Design. McGraw-Hill, New York.

17. Wide, L., E. Johannisson, K. G. Tillinger, and E. Diczfalusy. 1968. Metabolic clearance of human chorionic gonadotropin administered to nonpregnant women. Acta Endocrinol. 59:579-594.

18. Ashwell, G., and J. Harford. 1982. Carbohydrate-specific receptors of the liver. Annu. Rev. Biochem. 51:531-554.

19. Wehmann, R. E., and B. C. Nisula. 1979. Metabolic clearance rates of the subunits of human chorionic gonadotropin in man. J. Clin. Endocrinol. Metab. 48:753-759.

20. Sowers, J. R., A. E. Pekary, J. M. Hershman, M. Kanter, and J. J. DiStefano, III. 1979. Metabolism of exogenous human chorionic gonadotropin in man. J. Endocrinol. 80:83-89.

21. Rosa, C., S. Amr, S. Birken, R. Wehmann, and B. Nisula. 1984. Effect of desialylation of human chorionic gonadotropin on its metabolic clearance rate in humans. J. Clin. Endocrinol. Metab. 59:1215-1219. 